\title{
Los emprendimientos verdes una alternativa de sostenibilidad para las pymes en la ciudad de Cuenca.
}

Green entrepreneurship a sustainability alternative for SMEs in the city of Cuenca.

Iván Darío Plaza León. ${ }^{1}$, Christian Mauricio Banegas Campoverde. ${ }^{2}$ \& Yonimiler Castillo Ortega. ${ }^{3}$

DOI: https://doi.org/10.33262/visionariodigital.v5i2.1633

\begin{abstract}
.
Introduction. Green entrepreneurship is understood in this document, as the development of activities aimed at mitigating, reducing or eliminating environmental problems. It is observed the behavior between the human being with the environment in which changes are proposed within the production and consumption that harm the natural conditions of the planet. Objective. Design a management model based on the principles of green enterprises as a sustainability alternative for SMEs in the city of Cuenca. Methodology. This research work is descriptive and transversal in nature. To diagnose the management model of SMEs, a questionnaire structured in five principles of green enterprises was used: low-carbon economy, protection, transparency, knowledge and alliances. The reliability analysis of the questionnaire presented a Cronbach's Alpha coefficient of 0.924. Results. The results show that the actions of Cuenca's SMEs in terms of their management do not guide the development of their activities to mitigate, reduce or eliminate environmental problems related to production and consumption patterns that deteriorate the natural conditions of the planet. Conclusion. The components of a management model are established that incorporates in its scheme the principles of green enterprises, which allows the development of a culture of sustainability in SMEs in the

\footnotetext{
${ }^{1}$ Universidad Católica de Cuenca, Posgrado, Maestría en Desarrollo Local Mención en Ordenamiento Territorial, Cuenca, Ecuador, dariopla222@yahoo.com, https://orcid.org/0000-0002-0347-0206

${ }^{2}$ Universidad Católica de Cuenca, Carrera de Economía), Cuenca, Ecuador, cbanegas@ucacue.edu.ec, https://orcid.org/0000-0003-1102-2283.

${ }^{3}$ Universidad Católica de Cuenca, Posgrado, Maestría en Desarrollo Local Mención en Ordenamiento Territorial, Cuenca, Ecuador, ycastilloo@ucacue.edu.ec, https://orcid.org/0000-0002-7710-5199
} 
city of Cuenca, to create economic, environmental and social value, and make their management responsible action with the environment and society.

Keywords: Green entrepreneurship, sustainability, SMEs and sustainable companies.

\section{Resumen.}

Introducción. El emprendimiento verde es comprendido en este documento, como el desarrollo de actividades orientadas a la mitigación, reducción o eliminación de problemáticas ambientales. Se observa el comportamiento entre el ser humano con el entorno en el cual se proponen cambios dentro de la producción y el consumo que perjudican las condiciones naturales del planeta. Objetivo. Diseñar un modelo de gestión basado en los principios de los emprendimientos verdes como alternativa de sostenibilidad para las Pymes de la ciudad de Cuenca. Metodología. El presente trabajo de investigación es de naturaleza descriptiva y transversal. Para diagnosticar el modelo de gestión de las Pymes, se empleó un cuestionario estructurado en cinco principios de emprendimientos verdes: economía baja en carbono, protección, transparencia, conocimiento y alianzas. El análisis de fiabilidad del cuestionario presentó un coeficiente de Alpha de Cronbach de 0.924. Resultados. Los resultados evidencian que la actuación de las Pymes cuencanas en cuanto a su gestión, no orientan el desarrollo de sus actividades a mitigar, reducir o eliminar problemáticas ambientales, relacionadas con los patrones de producción y consumo que deterioran las condiciones naturales del planeta. Conclusión. Se establece los componentes de un modelo de gestión que incorpora en su esquema los principios de los emprendimientos verdes, que permita desarrollar una cultura de sostenibilidad en las Pymes de la ciudad de Cuenca, para crear valor económico, medioambiental y social, y hacer de su gestión una acción responsable con el medio ambiente y la sociedad.

Palabras claves: Emprendimiento verde, sostenibilidad, pymes y empresas sostenibles.

\section{Introducción.}

Históricamente se asocia la empresa y el emprendimiento a los objetivos de generación de riqueza, la creación de fuentes de empleo y el aumento en la competitividad de los mercados globales; además, se ha agregado la responsabilidad social y la reducción del impacto ambiental de las actividades empresariales (Kuckertz \& Wagner, 2010; Zahedi \& Otterpohla, 2015; Kurtagić, Silajdzic, \& Vucijak, 2015). Todo esto muestra que al hablar de emprendimiento según Schumpeter (1935), manifiesta y hace referencia a los individuos (emprendedores) quienes revolucionan el mercado con productos innovadores y novedosos.

El término emprendedor proviene del francés entrepernar (pionero), utilizado para referirse a los que se arriesgan a manifestar sus ideas a todo un mundo, así como de las oportunidades que existen sin saber cuál sea su futuro en el campo de su desempeño (Recalde, Villota \& Flores, 2016). Esta no es la única definición, pues según Pereira 
(2003), expuesto por (Chaves \& Castro, 2015), señala que el término entrepreneur surgió por primera vez en el contenido "Essai Sur la Nature du Commerce en General", escrito por Cantillon en 1755. Este concepto según el mismo autor fue perfeccionándose por aportes de Knight (1921), Baumol (1968 y 1993), Braudel (1985) y Casson (1982), donde diferenciaron entre un inversionista y un emprendedor, el primero espera el rendimiento sobre el capital mientas que el segundo busca el resultado de quitar de las utilidades que genera su emprendimiento los costos incurridos por el uso del capital del inversionista.

Para Dolabela (2010), todos somos emprendedores e incluso se nace emprendedor, pero es la estimulación que se reciba a lo largo de los años lo que determinará si se desarrollará o no esa característica. En consecuencia, cualquiera que reúna las características adecuadas estaría prácticamente predestinado a ser empresario (emprendimiento) y el serlo estaría en función del contexto que le rodee y la influencia de factores culturales, económicos, sociológicos y psicológicos. Concluyendo que «ser emprendedor es más que una cualidad, una actitud, un estilo de vida que va más allá de la creación de una empresa. Se puede ser emprendedor desde los diferentes ámbitos, tales como: social, cultural, deportivo y político (Yepes, 2012).

Moreno \& Olmos (2010) plantean mediante una investigación, que según un estudio realizado por Hisrich, Peters y Dean (2005) explican al emprendimiento desde los siguientes enfoques:

- Desde un enfoque del comportamiento: es la persona que toma la iniciativa, la cual consiste que puede organizar ideas en algo concreto, en un negocio y se asumen riesgos tras esa iniciativa.

- Desde un enfoque economista: es aquella persona que invierte los recursos necesarios, estos sean materiales, monetarios para poder incrementar su nivel económico.

- Desde un enfoque psicológico: es la persona por la que está motivada e inspirada a tener o cumplir un objetivo, la capacidad de poder obtener algo y no depender de nadie.

- Desde un enfoque generalista: es aquella persona que reúne los puntos de vistas anteriores. Es aquella persona que realiza una iniciativa con un valor, es decir dedicando perseverancia y además dedicar el tiempo que se requiere para el emprendimiento.

Considerando lo anterior, se puede asociar datos que arroja el reporte del Global Entrepreneurship Monitor (GEM) - Ecuador 2019-2020, sobre las Tasas de emprendimiento para el caso del Ecuador, donde la persona ecuatoriana tiene un comportamiento de asumir riegos al emprender en el área (tecnológico, empresarial, comercial, de servicios, industrial, ecológico). Es decir, que este año (2020) la Tasa de Actividad Emprendedora Temprana (TEA) es de 36,2\%, es decir que alrededor de 3.6 millones de habitantes estuvieron involucrados en la puesta de un emprendimiento, esta tasa varia con respecto al 2017 ya que se incrementó en 6.58 puntos porcentuales, quedando este año en el segundo lugar a nivel regional. (Lasio, Amaya, Zambrano, \& 
Ordeñana, 2020). Y finalmente, se puede acotar que aproximadamente unos de cada tres adultos ecuatorianos se han arriesgado por un negocio y lo han puesto en marcha.

\section{Emprendimientos verdes}

Hoy en día, el gran problema que existe en el mundo, son las practicas depredadoras que están causadas por un modelo económico antiguo de emprendimientos tradicionales, que se encarga del consumo de bienes y servicios al modo de satisfacer las necesidades del ser humano (Hancioglu, Uslu, \& Demir, 2015); por lo general no se toma en cuenta el gran impacto que ocasiona sobre los recursos no renovales que se consumen por estas actividades (Zoninsein, Cesano, y Russell, 2014). La gran dificultad es de observar a la naturaleza que es limitada, como factor de producción de bienes y servicios y por la cual se ha llegado hasta el punto de saturación sin retorno. (Vazquez, Smith , \& Sarkis, 2014; Zahedi \& Otterpohl, 2015).

En este sentido, viendo la crisis ecológica que afecta al mundo y a las empresas, sobresale un concepto que se denomina emprendimiento verde que según Dean \& McMullen, 2007; Ojeda \& Rodríguez, 2011, tiene sus origenes en la decada de los 90 y lo consideran como un concepto nuevo que ha ido en aumento, consiste en investigar, determinar y aprovechar las oportunidades que se presenten como fallas de mercado o de la industria entorno a actividades o situaciones sostenibles que sean rentables encadenadas al medio ambiente. Cuenta con dos directrices, la primera es que un emprendedor verde transforma cualquier línea de negocio y la convierte en un negocio verde, es decir eco-amigable; segundo que los empresarios tienen una orientación a favor de la protección del medio ambiente, es decir negocios constituidos como eco-amigables desde su creación (Sanabria \& Hurtado, 2010). Además, se puede considerar que se adapta a cualquier tipo de negocios, los cuales integran una economía sostenible en su plan de éxito (Nicuesa, 2016).

Por lo general, este tipo de emprendimientos se los puede llamar de diferentes nombres como: emprendimientos de base ecológica (Delgadillo \& Alburquerque, 2010), ecoemprendimiento (Gunawan, 2014), emprendimiento sostenible (Kuckertz \& Wagner; 2010), emprendimiento ecológico (OECD, 2011) y emprendimiento sostenible y ambiental (Dean \& McMullen, 2007).

No obstante estos emprendimientos, pueden generar negocios relacionados con la economia verde y la inversion responsable, para lograr un desarrollo sostenible dentro de las empresas (ComunicarSe,2018). En concordancia con lo anterior se mencionan 5 principios que son importantes para que una empresa se destaque de las demás y se catalogue como emprendimiento verde:

- Economía Baja en Carbono: Adoptar voluntariamente nuevas prácticas de negocio que contribuyan con la transición hacia una economía baja en carbono;

- Protección: Apoyar inversiones, actividades y proyectos que tengan un impacto ambiental y social positivo y buenas prácticas de gobernanza;

- Transparencia: Divulgar reportes de sostenibilidad en las empresas; 
- Conocimiento: Crear y fortalecer las capacidades y conocimientos de diversos sectores, para lograr el desarrollo sostenible;

- Alianzas: Coordinar programas con el sector público, privado, la academia y la sociedad civil con el fin de acelerar el desarrollo de la económica Verde.

A más de los principios verdes se estudian las características, que según la Escuela Europea de Excelencia (2019), establecen que las empresas que se consideren verdes y que generen un mayor impacto dentro de la sociedad y el medio ambiente, deben tener ciertas caracteristicas como: Optimización de recursos, reducción de contaminación, eliminación de desperdicios, tener un producto más competitivo, reducción de costos, un valor agregado socio-ambiental, mejora las condiciones de vida, oferta una solución tecnológica, por el perfil que se requiere, los empleos tienen un mayor nivel de ingresos, mejor nivel de vida, mitigan el cambio climático, reducen la contaminación y maximizan la eficiencia a través de un servicio o producto.

\section{Sostenibilidad empresarial}

Elkington (1999), menciona que la sostenibilidad desde un principio ha sido difícil de integrar, ya que las necesidades son diversas con respecto al desarrollo del ser humano, es decir, a lo largo del tiempo se viene dando un debate ya que solo este término se encuentra escrito mas no se aplica dentro de la sociedad. Durante la historia se han llevado a cabo algunos acciones y acuerdos sobre el desarrollo sostenible como: la conferencia mundial realizada en Estocolmo en 1972 donde el resultado es que se crea el Programa de las Naciones Unidas para el Medio Ambiente (PNUMA) y la cumbre sobre el cambio climático en París en 2015 donde se llegan a acuerdos en financiamiento para afrontar el cambio climático, urbanismo, industria, energías renovables y transporte alternativo eléctrico. Todo esto se da en el ámbito de integrar a todos los involucrados como los gobiernos, instituciones internacionales, empresas, sociedad y medio ambiente (Manuel, 2015).

Por otra parte, Barcellos (2010), cita que la sostenibilidad empresarial no es más que un concepto complejo que no puede solucionarse en una sola acción corporativa, se tiene que trabajar por parte de las empresas en minimizar los residuos de sus operaciones y al mismo tiempo la reorientación de su cartera de competencias hacia tecnologías limpias. Para ello se cuenta con 3 pilares fundamentales para que el modelo sostenible tenga éxito dentro de la empresa: la estrategia, gestión y comunicación, donde la primera consiste en construir una hoja de ruta en la cual se determina hasta donde se puede llegar la empresa, la segunda consiste en la puesta en acción en todas las actividades que realice dentro de la empresa tanto en sus estrategias como de la propia gestión, y por último se establece la comunicación donde se debe contar una bonita historia donde debe prevalecer la trasparencia hacia la comunidad (Amate, 2015).

Para lograr una sostenibilidad empresarial que afronte los cambios en su entorno durante el largo plazo, se debe plantear estrategias que incluyan 5 factores claves: principios organizacionales, fortalecimiento social, compromiso de la dirección, informes de 
progreso y acción local, todo esto se implementara sin importar el tamaño de la empresa, la ubicación o el tipo de constitución legal (Koga, 2016).

\section{Los modelos de gestión basados en emprendimientos verdes}

Un modelo de gestión, como lo explica Serna (2002) es la forma de planificar organizar, dirigir y controlar las acciones de la empresa. A su vez, este debe hacer hincapié en las relaciones de contexto con el medio externo como interno, donde la primera se trata de aprovisionar insumos, recursos humanos, clientes, mientras que la segunda debe considerar tres enfoques, que son: los roles administrativos, la toma de decisiones y los sistemas dinámicos (Simón, 2005).

Autores como Arroyo et al (2018), considera que la innovación del modelo empresarial verde, se debe realizar de una manera continua para generar efectos ecológicos y económicos dentro del negocio. Por lo que en la actualidad varias empresas han implementado algunos métodos y estrategias tales como:

- Los Modelos Incentivos: Es la forma de dar incentivos a los consumidores por medio del cambio de la cadena de valor, dando un cambio de lo tradicional a lo ecológico como por ejemplo las empresas empresa de ahorro de energía, servicio de gestión de sustancias químicas, y finalmente el modelo diseñar, construir, financiar, operar.

- Modelos del Ciclo de Vida: Nos permite a no perder la perspectiva ambiental, social, cultural, al momento de diseñar productos o procesos pudiendo prever y evitar los posibles impactos. Dentro de este modelo se puede hablar del modelo cuna a cuna, que no es más que diseñar nuevos productos y procesos en un sistema que adopte una estrategia para el cambio más allá de la eco-eficiencia, tomando como fuente de inspiración los sistemas naturales, además existe una certificación (Cradle to Cradle ${ }^{\mathrm{TM}} \mathrm{C} 2 \mathrm{C}$ ), que involucra a todas las industrias que reutilizan varias veces sus productos y lo convierten en productos que pueden volver a la cadena de producción como también a la tierra en nutrientes biológicos.

En el marco de modelo de gestión empresarial de los negocios verdes inclusivos, según la CAF (2015) es un diseño que cuenta con asesoría y financiamiento por parte de esta institución, a su vez está orientada hacia la implantación de un sistema de gestión empresarial que a la vez que fomenta la inclusión social y el uso sostenible de la biodiversidad, fomenta y articula el mercado, fortalece la institucionalidad y educa al consumidor local. Cuenta con un enfoque de inclusión social por medio de la protección y conservación del ambiente a través de actividades financieramente rentables.

Para este tipo de modelo se toman empresas que existen en el mercado, y por medio de la aplicación de patrones de ecoeficiencia o la búsqueda de certificaciones como por ejemplo la ISO 14001, genera y se convierten cualitativamente en empresas sostenibles ambientalmente y cuentan con una mayor rentabilidad y productividad en todas sus 
operaciones. Con este antecedente se puede presentar un esquema que se reparte por etapas (componentes) para el desarrollo y consolidación del proyecto en función de sistemas de planificación de inversión público-privada, que estén sincronizados con ciclos de financiamiento, se toma como referencia el modelo que nos presenta la CAF con sus respectivas etapas:

\section{Etapa 1 Entorno Nacional (Identificación)}

- Socios estratégicos locales

- Parámetros de operación

- Capacitación y entrenamiento de asesores técnicos

- Elaboración de guía local de negocios verdes

- Propuesta de marco legal para impulsar emprendimientos verdes en Panamá

\section{Etapa 2 Divulgación, Sensibilización y Diagnóstico (Diseño)}

- Lanzamiento

- 4 conferencias de difusión

- 15 seminarios / talleres de sensibilización

- Campañas de medios: brochure, redes sociales y sitio WEB

- Diagnóstico empresarial Acompañamiento técnico

- Evaluación y selección de proyectos, rechazo o aceptación diferida

- Capacitación de fortalecimiento empresarial y técnico

\section{Etapa 3 Desembolsos (Ejecución)}

- Primer desembolso: arranques y/o mejoras

- Segundo desembolso: plan de negocios y sostenibilidad del emprendimiento

- Tercer desembolso (último desembolso): evaluación independiente por socios estratégicos.

\section{Etapa 4 Evaluación Final}

- Visitas de campo y compilación de lecciones aprendidas

- Auditorías financieras a todos los niveles

Otro Modelo de gestión que se utiliza dentro de las micro y pequeñas empresas, se trata de una matriz de marco lógico que se pueden aplicar en proyectos nuevos o en marcha. Es decir, es una propuesta que se puede implementar como un plan piloto que servirá de un modelo de gestión de negocios verdes para la región. (CAF, 2015). Este modelo cuenta con las mismas etapas que se trataron en el modelo de gestión empresarial de los negocios verdes inclusivos.

Se aborda a continuación, algunos casos de modelos exitosos en temas de emprendimientos e innovación que se han ejecutado a nivel internacional en empresas existentes y nuevas creadas como verdes.

Según Salgado ( 2012) en el Tabla 1 plantea desde un punto de vista de la RSE dentro de la empresa, donde la función de trabajo es a favor de los diferentes grupos de interés con 
los que interactúa sobre los impactos que genera debido a su actividad, como de comunicar y ver la manera de implementar dentro de su organización de la mejor manera.

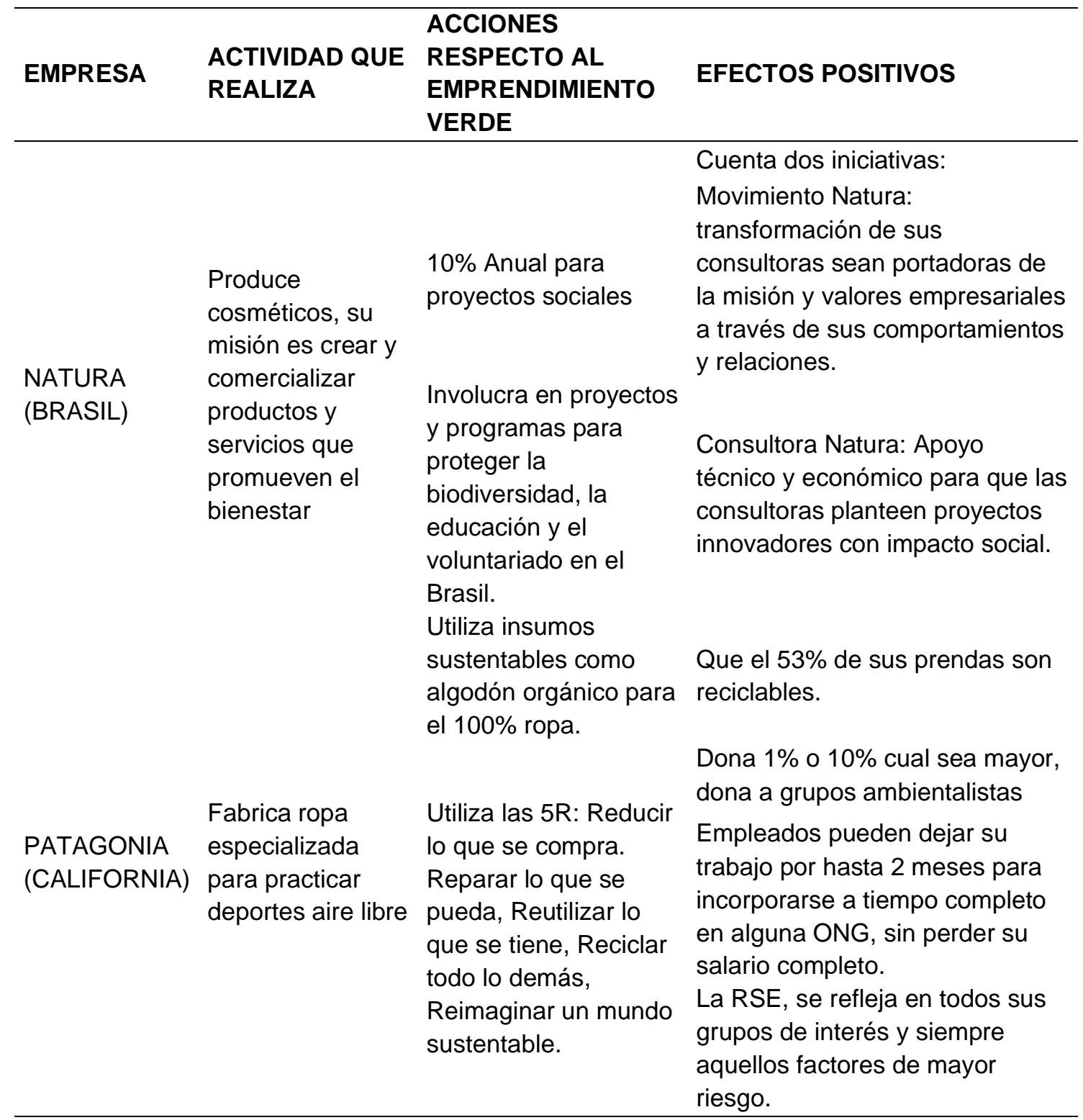

Tabla 1. Emprendimientos verdes existentes Fuente: (EKOS, 2012)

Analizando otros emprendimientos exitosos que fueron creados y que se detallan en la Tabla 2.

\begin{tabular}{|c|c|c|c|c|}
\hline EMPRESA & $\begin{array}{l}\text { ACTIVIDAD } \\
\text { QUE REALIZA }\end{array}$ & $\begin{array}{l}\text { COMPONENTES } \\
\text { RESPECTO AL } \\
\text { EMPRENDIMIENT } \\
\text { O VERDE }\end{array}$ & EFECTOS POSITIVOS & FUENTE \\
\hline $\begin{array}{l}\text { GROCOL } \\
\text { (COLOMBIA) }\end{array}$ & $\begin{array}{l}\text { Desarrollo de } \\
\text { proyectos } \\
\text { sostenibles } \\
\text { para el sector } \\
\text { de la }\end{array}$ & $\begin{array}{l}\text { Tener un impacto } \\
\text { significativo sobre el } \\
\text { medio ambiente. }\end{array}$ & $\begin{array}{l}\text { Proceso de } 572 \text { toneladas } \\
\text { de basura orgánica se han } \\
\text { convertido en un sustrato } \\
\text { especializado para el } \\
\text { crecimiento de las plantas. }\end{array}$ & \\
\hline
\end{tabular}


Vol. 5, $\mathrm{N}^{\circ}$ 2, p. 6-29, abril-junio, 2021

construcción:

techos y muros

verdes, ha

construido más

de 180

proyectos $\mathrm{y}$

más de

150.000 metros

cuadrados de

infraestructura

vegetada

URBAN BIKE

(Bilbao) venta, alquiler

de Bicicletas;

así como

imparten

cursos de

como pedalear.
Precios razonables

para producir

beneficios

económicos para

nuestros clientes.

Mejorar la calidad

de vida de las

personas.

Ser técnicamente viables y garantizar una estabilidad de los proyectos en el largo plazo.

Apuesta por su calidad y la especialización.

Contar con

bicicletas urbanas

como de montaña o

eléctricas

Captar al cliente

adicionando algo

extra (alforjas

Ortlieb).
Techos han capturado más de 2,8 millones de litros de agua evitando que vayan a

los saturados

(Groncol, alcantarillados que

tenemos en este país y

posteriormente a los

contaminados ríos. Esto

genera un ciclo limpio del

agua y es el equivalente a

62.000 duchas.

Compensan la huella de

carbono de 10.300

personas, y el material

articulado emitido por

7.700 carros.

Disminución en la

contaminación dentro de la

Ciudad.

Creación de un colectivo

(Crespo, de ciclistas, por lo que se

2018) los automovilistas.

\section{Tabla 2. Emprendimientos verdes creados}

\section{Fuente: Elaboración propia.}

Como se puede observar en la Tabla 1 y 2 , se destacan algunos ejemplos exitosos que cuentan con acciones y componentes que las empresas aplican para alcanzar los efectos positivos que beneficiaran tanto a las empresas, personal, sociedad, medio ambiente; y mediante estos casos de estudio se pueda medir los emprendimientos verdes que puedan generar relaciones locales sostenibles.

Por tanto, la presente investigación pretende contribuir a que las Pymes, puedan tener un modelo de gestión para que puedan implementar dentro de sus empresas, así como para los nuevos emprendimientos que requieran contribuir al cuidado del medio ambiente, generar nuevos empleos y dinamizar la economía del país, y sobre todo en la ciudad de Cuenca.

\section{Metodologia.}

La presente investigación es de naturaleza descriptiva y transversal. a) Descriptiva, debido a que se identificó las características de los elementos de las empresas que cumplen con ciertos rasgos importantes sobre el emprendimiento verde en la ciudad de 
Cuenca. b) Transversal, puesto que se pretende analizar datos recolectados a través de un instrumento estructurado, en un único momento en el tiempo.

\section{Instrumento de recolección de datos}

Para la presente investigación se tomó la información necesaria, la cual fue adquirida mediante la técnica de la encuesta, por lo que se utilizó como instrumento de recolección de datos un cuestionario de 46 preguntas. El cuestionario es formal y estructurado, consta de tres partes. La primera y segunda son secciones preliminares de carácter informativo que recopila información de las Pymes y de la persona que brindó la información de la empresa, consta de 7 preguntas. La tercera sección consta de cuestionamientos dirigidos a obtener información sobre el modelo de gestión de las Pymes de la ciudad de Cuenca, considerando cinco principios: Economía baja en carbono, Protección, Transparencia, Conocimiento y Alianzas, con un total de 37 preguntas. Y en la última sección, se aplicaron 2 preguntas de criterio personal con respecto a ¿cómo se imaginan en 5 años si aplican un modelo de gestión en base a emprendimientos verdes? y finalmente, según su opinión ¿Cuáles medidas, acciones, programas o leyes se deberían modificar o implementar para fomentar el emprendimiento verde en la ciudad de Cuenca?

El cuestionario que se utilizo fue aplicado a las Pymes cuencanas, en el cual, las preguntas se presentan en forma de afirmaciones en una escala de tipo nominal con dos opciones de respuesta (dicotómicas), con un valor numérico que se representa de la siguiente forma: Si (1) y No (2).

Para la presentación e interpretación de los resultados se realizó un descriptivo para conocer sobre las características que presenta el modelo de gestión de las Pymes de la ciudad de Cuenca. Se obtuvo la media por principio, mediante la fórmula $\frac{\sum x_{i}}{n}$ (donde $\mathrm{x}_{\mathrm{i}}$ son los valores porcentuales de cada pregunta y n es el número total de preguntas del principio), y estos datos se presentaron mediante gráficos estadísticos.

La fiabilidad del cuestionario acorde a lo que Kline citado por Tejada (2004), donde menciona "que los coeficientes de confiabilidad de alrededor de 0.90 se consideran excelentes, valores alrededor de 0.80 como muy buenos, valores alrededor de 0.70 , como adecuados y aquellos menores de 0.50 indican que al menos la mitad de la varianza observada puede ser debida a error al azar, y medidas tan poco confiables deberían ser evitadas", es decir aquí se mide el grado fiabilidad del cuestionario lo cual se utilizó el programa SPSS, donde se calculó el coeficiente de Alfa de Cronbach, y cuyo valor que se obtuvo es de 0.924 ; lo que significa, que el cuestionario que se aplicó tiene fiabilidad,

\section{Universo y toma muestral}

La población está definida por la cantidad de empresas que se encuentran registradas en el INEC y que desempeñan la actividad comercial según código CIIU 4.0. La base del 
Instituto Nacional de Estadística y Censos (INEC) proyecta 3975 empresas las cuales serán definidas como población objeto de estudio.

El cálculo de la muestra fue mediante el método probabilístico utilizando la varianza máxima (p x q a un valor máximo de 0,5), con un nivel de confianza de $95 \%$ y un error máximo admisible de $5 \%$. Estos valores fueron aplicados mediante la fórmula de Murray y Larry (2005), para el cálculo de la muestra, y se utilizó los datos de todo el universo de Pymes que existen dentro de la ciudad de Cuenca, que es un universo finito.

Por lo tanto, la muestra del presente estudio quedó constituida por un total de 351 Pymes de la ciudad de Cuenca, de las cuales el $63.7 \%$ de las empresas tienen más de 10 años de creación y el 36.3\% tienen entre 1 y 9 años de creación. En relación al número de empresas por su tamaño se tiene que el $84 \%$ son pequeñas empresas, el $12.6 \%$ son medianas tipo "B" y el 3.4\% son medianas tipo "A", estas categorías se establecieron según la Comunidad Andina de Naciones (CAN). En consecuencia, a lo antes mencionado se puede detallar las actividades económicas dentro de las Pymes de la ciudad de Cuenca donde el sector económico más fuerte es el de: servicios con un 38.8\%, Industrias manufactureras $32 \%$, Comercio $21 \%$, Construcción $6 \%$ y dejando por último Agricultura, ganadería, silvicultura, pesca y Explotación de minas, canteras con tan solo el $2.1 \%$ y $0.1 \%$ respectivamente.

\section{Resultados.}

Los datos fueron analizados de acuerdo a los principios que se mencionaron anteriormente y que se refleja en la Tabla 3, donde cada una de éstos consta de un número determinado de ítems vinculados al modelo de gestión de las Pymes.

Estos principios que son analizados, se modificaron en el cuestionario de acuerdo a la realidad de las Pymes de la ciudad de Cuenca.

\begin{tabular}{lc}
\hline \multicolumn{1}{c}{ Principio } & Ítems \\
\hline Economía baja en carbono & $1-13$ \\
Protección & $14-24$ \\
Transparencia & $25-26$ \\
Conocimiento & $27-33$ \\
Alianzas & $34-37$ \\
\hline \multicolumn{2}{c}{ Tabla 3. Principios utilizados para el análisis } \\
\multicolumn{2}{c}{ Fuente: Cuestionario. }
\end{tabular}

A continuación, se presenta los resultados de la valoración de los principios:

\section{Principio 1: Economía baja en carbono}

Es este apartado se puede analizar este principio como un proceso de transición a una economía con bajas emisiones de carbono, en base a examinar la dimensión cualitativa y 
cuantitativa de los empleos de manera justa hasta llegar a la sostenibilidad de las empresas. (Torre, 2018)

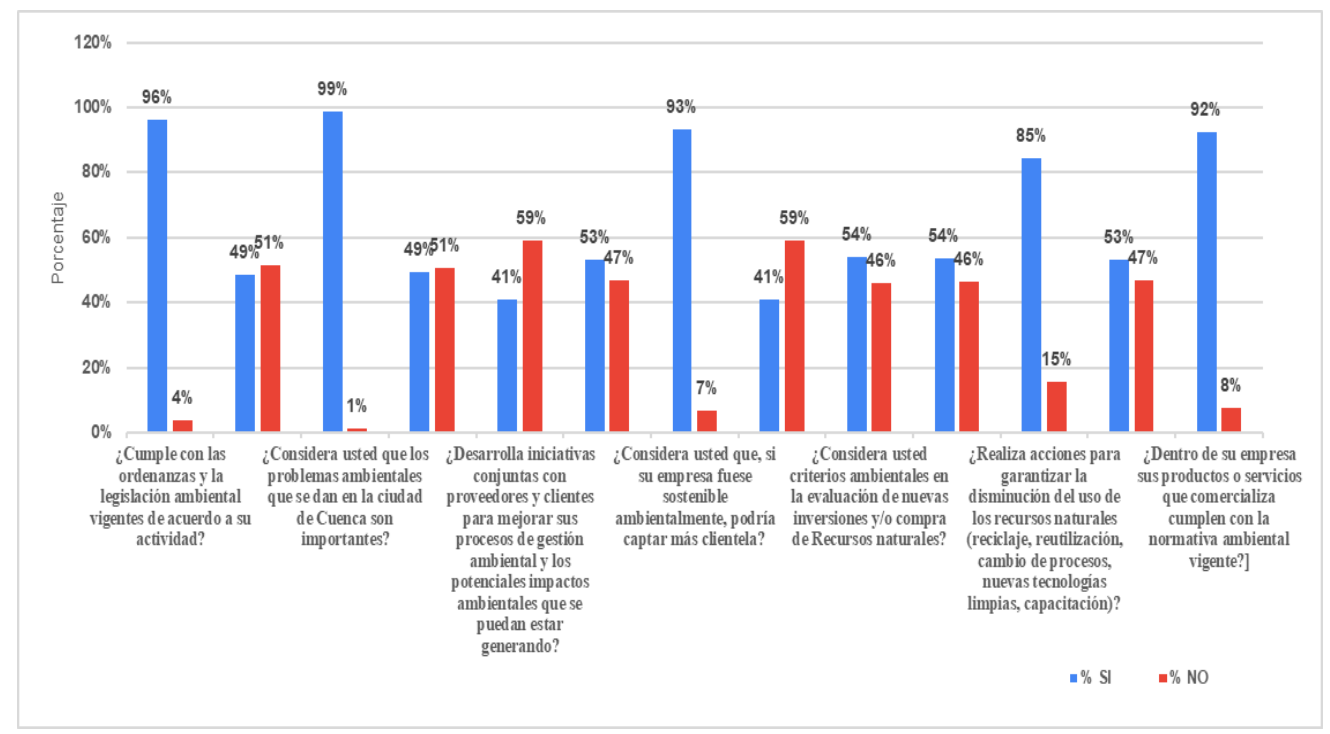

Figura 1. Diagnóstico del principio Economía baja en carbono Fuente: Cuestionario.

En la Figura 1, se muestra que el $99 \%$ de los problemas ambientales que se dan en la ciudad de Cuenca son importantes, y que un $92 \%$ de las empresas cumplen con la normativa vigente en la comercialización de sus productos y servicios que brindan a la población en general, mientras que un 59\% no cumple con un plan de contingencia en caso de ocurrir un problema ambiental dentro de su negocio.

Este principio fue evaluado con 13 ítems, donde se obtuvo un valor promedio del $66 \%$ de respuestas positivas $(\mathrm{Si})$ y un $34 \%$ de respuestas negativas (No). Lo que significa, que más de la mitad de las empresas encuestadas consideran que es de vital importancia adoptar de manera voluntaria nuevas prácticas de cuidado del medio ambiente que permitan contribuir a una economía baja en carbono.

\section{Principio 2: Protección}

Se refiere a que se debe apoyar a inversiones que se realicen, actividades y proyectos que tengan un impacto ambiental y social positivo y buenas prácticas de gobernanza (ComunicarSe, 2018). 


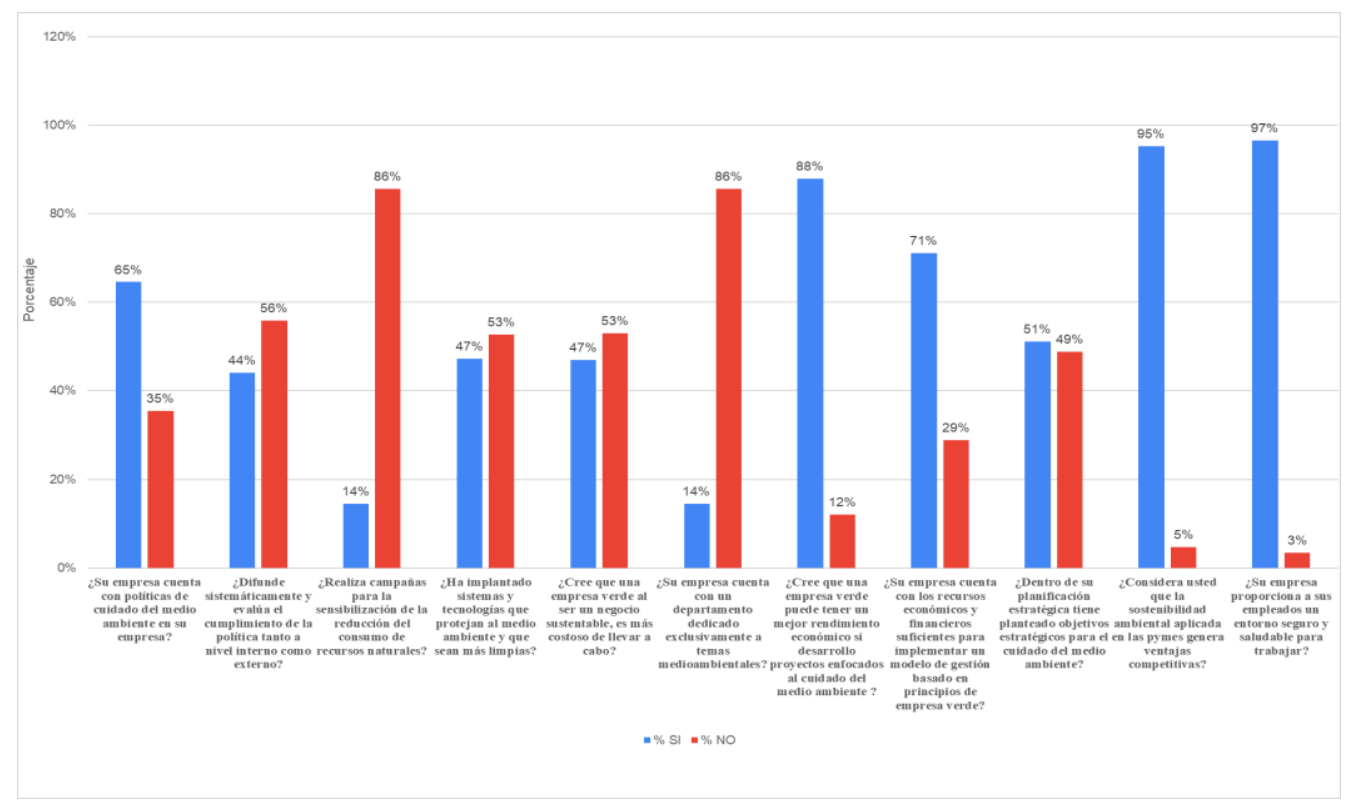

Figura 2. Diagnóstico del principio Protección Fuente: Cuestionario.

En la Figura 2, se puede ver qué aspectos que pueden rescatarse, por ejemplo, el 51\%, $65 \%$ y $71 \%$ de los encuestados tienen claro que cuenta con políticas, buen rendimiento económico y cuenta con los recursos económicos y financieros suficientes para implementar un modelo de gestión basado en principios de empresa verde. Además, el $88 \%$ de los encuestados pueden tener éxito si desarrollan proyectos sobre el cuidado del medio ambiente dentro de su empresa, y el 95\% y 97\% les interesa que sus empresas generen ventajas competitivas si aplican modelos verdes y que a sus empleados están dentro de un ambiente laboral agradable pero que se puede mejorar.

Este principio fue evaluado con 11 ítems, donde se obtuvo un valor promedio del $58 \%$ de respuestas positivas $(\mathrm{Si})$ y un $42 \%$ de respuestas negativas (No). Lo que significa, que más de la mitad de las empresas encuestadas están trabajando para llevar y cumplir con este principio.

\section{Principio 3: Transparencia}

El principio de trasparencia de la información con respecto a las empresas, debe estar disponible para su consulta con datos claros, verificados y que deben ser entregados de forma voluntaria, sin esperar que se solicite por alguna entidad de control o norma vigente al respecto. (Ramírez, 2020) 


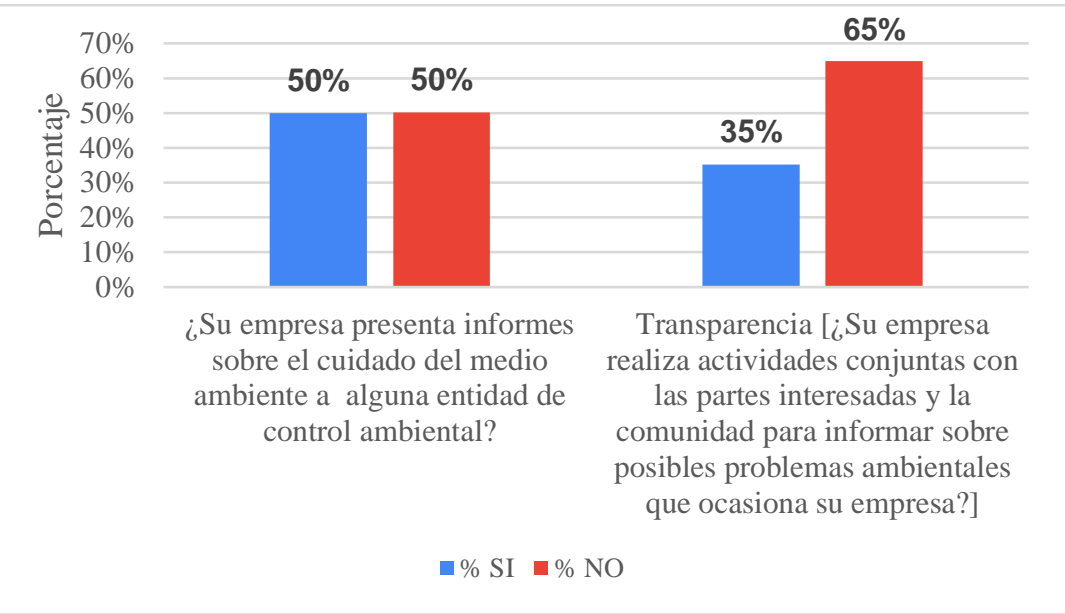

Figura 3. Diagnóstico del principio Transparencia Fuente: Cuestionario.

En la Figura 3, los resultados obtenidos se muestran en porcentajes que son iguales y menores al 50\% de la empresa encuestada, que responden que presentan algún informe sobre el cuidado del medio ambiente a alguna empresa de la ciudad (50\%), mientras que un $65 \%$ no realiza actividades conjuntas con las partes interesadas y la comunidad para informar sobre posibles problemas ambientales que ocasiona su empresa.

Este principio fue evaluado con 2 ítems, donde se obtuvo un valor promedio del $43 \%$ de respuestas positivas ( $\mathrm{Si}$ ) y un $57 \%$ de respuestas negativas (No). En consecuencia, se debe generar reportes o informes sobre el cuidado del medio ambiente y publicarlos para que la sociedad este actualizada sobre estos temas.

\section{Principio 4: Conocimiento}

Este principio debe estar dirigido para lograr un fin y es la suma de los conocimientos de sus empleados y otros valores, por ende, se suele decir que se radica en los procesos, clientes, el personal y es importante distinguir entre las fuentes de información y las de conocimiento. (Udiz, 2010)

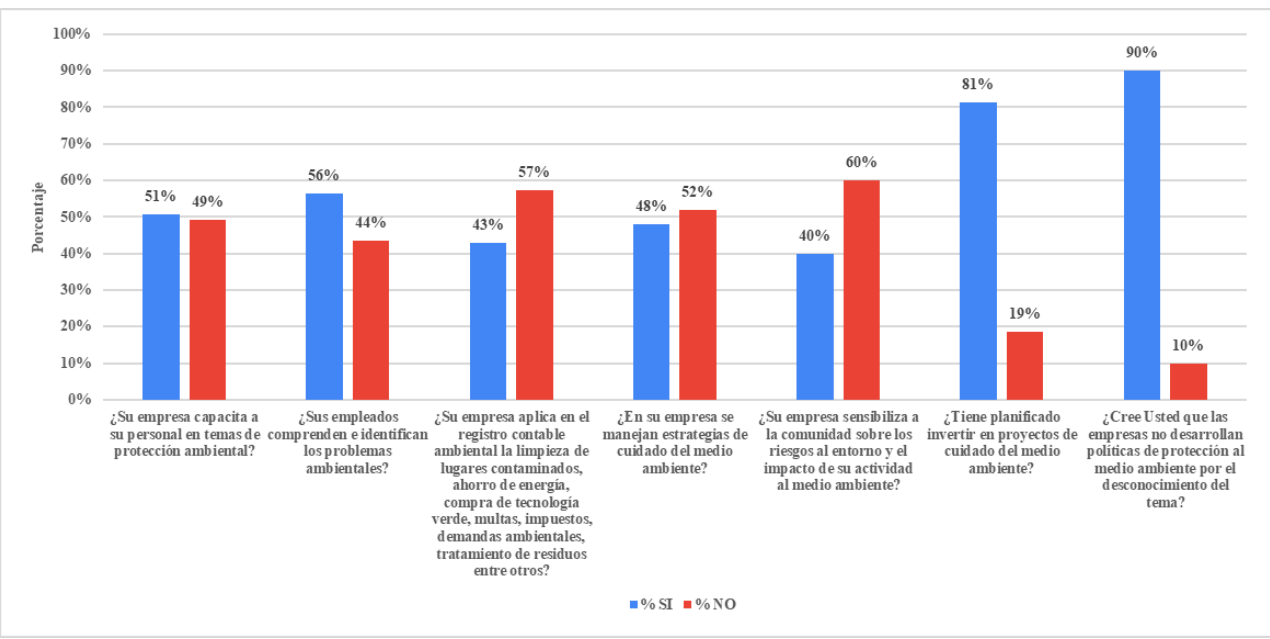

Figura 4. Diagnóstico del principio Conocimiento Fuente: Cuestionario. 
En la Figura 4, se puede resaltar que el 90\% de las empresas no dan mucha importancia al tema ambiental por desconocimiento del tema, pero al mismo tiempo un $81 \%$ tiene planificado en invertir en proyectos o emprendimientos que protejan el medio ambiente, adicionalmente, un $56 \%$ de sus empleados conocen sobre los problemas ambientales que se dan en la mayoría de las empresas y además un 51\% de las empresas capacita a su personal en estos temas.

Este principio fue evaluado con 7 ítems, donde se obtuvo un valor promedio del $58 \%$ de respuestas positivas ( $\mathrm{Si}$ ) y un $42 \%$ de respuestas negativas (No). Por lo general se debe mejorar en algunos aspectos como: llevar un registro contable sobre el ahorro de energía, compra de tecnología verde, multas, impuestos, demandas ambientales, tratamiento de residuos entre otros, además el no comunicar los riegos que ocasionaría si se produjera algún inconveniente dentro de sus instalaciones como fuera de ellas.

\section{Principio 5: Alianzas}

Este último principio es la resultante de analizar algunos aspectos como la planeación, voluntad, claridad y confidencialidad, estas deben estar en sinergia ya que al combinar estas se dan un contacto directo entre las empresas pública - privada y la universidad, para llegar a concretar alianzas de las cuales ganen las partes interesadas, siempre y cuando protejan el cuidado del medio ambiente. (Pérez, 2019)

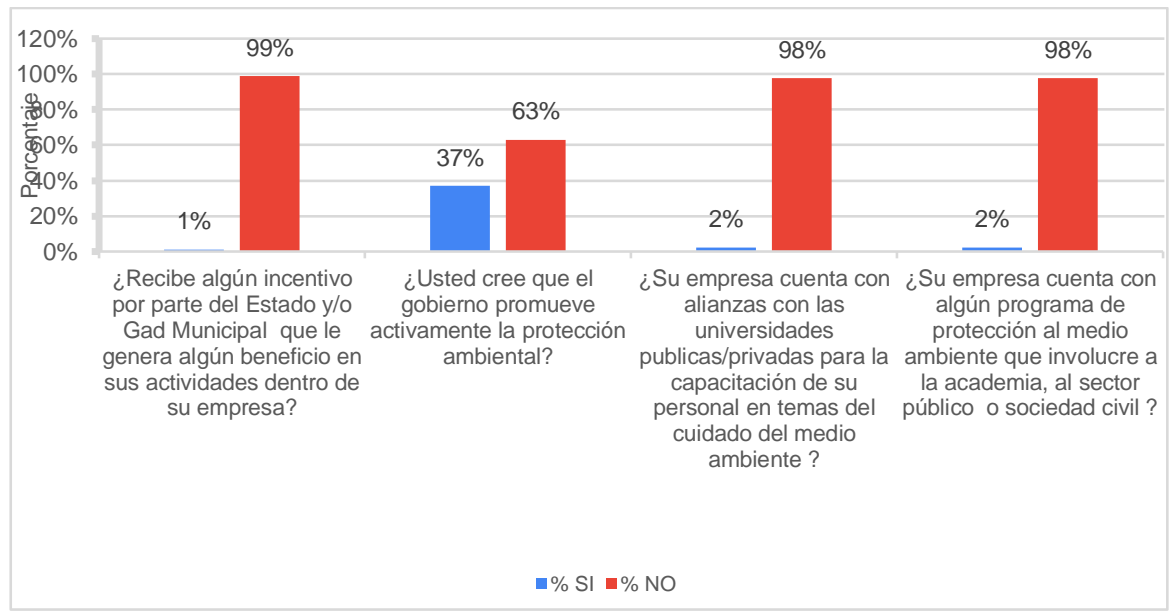

Figura 5. Diagnóstico del principio Alianzas Fuente: Cuestionario.

En la Figura 5, las respuestas que fueron respondidas por el personal de las empresas se tienen que un rango de 98 al 99\% afirman que no se ha logrado recibir algún incentivo por parte del gobierno central, así como existe un porcentaje alto que no ve el apoyo de la academia en atención y al cuidado del medio ambiente hacia las empresas cuencanas, es decir que apenas el $37 \%$ cree que el estado promueve activamente la protección ambiental.

En consecuencia, este principio fue evaluado con 4 ítems, donde se obtuvo un valor promedio del $11 \%$ de respuestas positivas ( $\mathrm{Si}$ ) y un $89 \%$ de respuestas negativas (No). Lo 
que significa, que se debe trabajar más a fondo en algunos aspectos como: integración entre lo público-privado.

Otro punto que se puede describir es que en base de los resultados aplicados en el cuestionario, se alcanzó a verificar que una pregunta que se les realizo que ¿ Cómo se imagina a su empresa en 5 años, si aplicaría un modelo de gestión basado en principios de emprendimiento verde?, se determinó que un $99 \%$ de las empresas encuestadas de la ciudad de Cuenca estarían en la posibilidad de aplicar un modelo que exista y que trate de mejorar su economía y sobre todo estarían aportando para el cuidado y protección del medio ambiente.

Como punto final se preguntó qué ¿Cuáles medidas, acciones, programas o leyes se deberían modificar o implementar para fomentar el emprendimiento verde en la ciudad de Cuenca?, las respuestas fueron que más del $50 \%$ se debería enfocar en ayudar con subsidios, incentivos, capacitación por parte del gobierno e instituciones públicasprivadas; todo esto será beneficioso para el cuidado del medio ambiente.

\section{Propuesta: Modelo de Gestión para emprendimientos verdes.}

Luego de analizar y establecer el marco teórico, se puede plantear el modelo a seguir para que las Pymes de la ciudad de Cuenca puedan encaminar sus empresas para que puedan transformarse en empresas verdes, este modelo de gestión se diseña mediante 2 aspectos que se detallan a continuación:

1. Para establecer el modelo de gestión para emprendimientos verdes se consideran las siguientes etapas: Identificación, diseño, ejecución y evaluación y seguimiento, el mismo que está tomado según la CAF 2015, el mismo que fue pensado para todas las Pymes cuencanas de acuerdo a sus actividades económicas que desempeñan, en la siguiente figura 6 se muestra el modelo.

2. Como segundo punto se determina que, dentro del modelo de gestión se incorpora los 5 principios de emprendimientos verdes que se explicaron en el marco teórico y que se ajustaron de acuerdo a la realidad de las Pymes cuencanas.

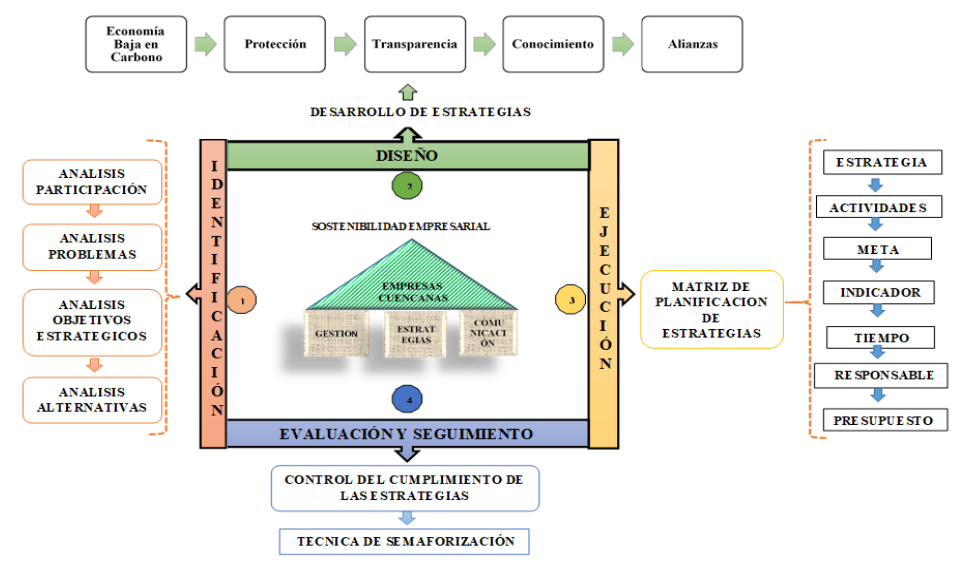

Figura 6. Modelo de gestión para emprendimientos verdes. Fuente: Elaboración propia. 


\section{Identificación}

Se caracteriza esta etapa en determinar el problema a resolver, una necesidad u oportunidad, es una introducción a la preparación y evaluación de un modelo a seguir. Sin embargo, se deben considerar algunos pasos antes de plantearse un modelo que esté basado en estrategias del cuidado del medio ambiente y la sostenibilidad empresarial:

1.1 Establecer el Análisis de la participación, donde se establecen quienes estarán involucrados directamente en las actividades empresariales, a estos se les puede llamar beneficiarios o perjudicados y se identifican internamente (propietarios y empleados) como externamente (proveedores, clientes, sociedad, gobierno y medio ambiente). Para esto las gestiones y medidas que se tomen deben ir encaminadas a mejorar el bienestar de los involucrados con una proyección a fomentar la sostenibilidad empresarial.

1.2 Análisis de los problemas que existe dentro de las empresas basándose en los 5 principios de economía verde, es decir, construir un mapa con gráficos en donde se encuentre el problema central, el mismo que nos servirá para ordenar las ideas que vayan surgiendo. Y este mapa nos servirá para ir generando las nuevas estrategias para construir un modelo de gestión que vaya de la mano con el cuidado del medio ambiente y la sostenibilidad empresarial.

1.3 Analizar los objetivos estratégicos, es decir, se debe restructurar estratégicamente los objetivos, la misión y su visión de la empresa, donde se puedan visualizar los propósitos de ser una empresa verde, por lo que muestren sus acciones frente a las personas, proveedores, gobierno y medioambiente. Con esta reestructuración las empresas se vuelven éticamente más responsables con el bien común y se configura una nueva filosofía de no solo conocer a una empresa que produzca bienes y servicios para el consumo humano, sino el plantear un nuevo horizonte del cuidado y protección del medio ambiente.

1.4 Analizar las alternativas que generarán o combatirán las deficiencias encontradas dentro de las empresas. Para lo cual se debe plantear algunas estrategias que más adelante se aplicaran dentro de los principios de economía verde. Se deben considerar, algunas cuestiones que son fundamentales para plantearse estrategias que vayan de acorde a las realidades de las empresas de la localidad: consumo sostenible de los recursos naturales renovables o bienes y servicios que de ellos se deriven, instrumentos que promuevan el financiamiento para los emprendimientos verdes y tomar en cuenta los riegos en los nuevos planes que se pretendan financiar.

\section{Diseño}

Se refiere esta etapa a armar o diseñar las estrategias a seguir para llegar a construir un modelo que deje a un lado las empresas tradicionales y las convierta en empresas verdes, para ello las estrategias estarán basadas en las problemáticas encontradas en la etapa 
anterior. Y en este apartado se deben considerar los 5 principios verdes tomados de ComunicarSe, 2018; y que servirán para armar las estrategias a seguir:

1) Economía Baja en Carbono:

- Conocer los permisos, licencias o fichas ambientales que otorguen el Gobierno Municipal u otra institución para funcionar.

- Contar con criterios para seleccionar proveedores verdes.

- Trabajar con nuevos insumos que cuiden el medio ambiente.

- Monitorear el consumo de energía, agua, otros recursos naturales y/o de materias primas no renovables.

- Adaptar el tema de reciclaje, reutilización, cambio de procesos, nuevas tecnologías limpias, capacitación.

- Incluir el tema verde en el desarrollo y comercialización de los nuevos productos o servicios.

2) Protección:

- Redactar nuevas políticas dentro de su empresa sobre el cuidado del medio ambiente y hacerlo conocer tanto a nivel interno como externo.

- Implementar nuevas tecnologías verdes, que protejan al personal y al medio ambiente.

- Implementar un departamento que se encargue en trabajar en proyectos que estén enfocados al cuidado del medio ambiente.

- Dotar de las herramientas necesarias a todo el personal de su empresa, para que puedan trabajar de una manera segura y saludable.

3) Transparencia:

- Entregar los respectivos informes sobre el cuidado que da su empresa con el medio ambiente, con esto se cumple el respeto hacia la naturaleza y la comunidad.

- Realizar actividades en conjunto a la comunidad en temas relacionados al cuidado y protección del medio ambiente, mediante campañas que expliquen lo que ocasiona una empresa que no esté en el tema verde.

4) Conocimiento:

- Implementar una serie de capacitaciones a todo el personal incluido el gerente de la empresa, para conocer a fondo los problemas que ocasionan el impacto de las empresas no verdes.

- Implementar un sistema contable ambiental, para que se pueda controlar algunos rubros como el: ahorro de energía, compra de tecnología verde, multas, impuestos, demandas ambientales, tratamiento de residuos entre otros.

5) Alianzas:

- Crear programas sobre la protección del medio ambiente, donde se incluyan a la: academia, al sector público o sociedad civil.

- Crear alianzas con las universidades publicas/privadas para la capacitación de su personal en temas del cuidado del medio ambiente. 
Todos estos aspectos encontrados pueden generar que las empresas cuencanas puedan seguir un camino hacia la sostenibilidad empresarial y el cuidado del medio ambiente.

\section{Ejecución}

Esta etapa consiste en la ejecución del diseño del modelo a seguir desarrollado en la etapa anterior, para lo cual se deben definir una ruta hacia el camino de la sostenibilidad y empresas verdes. Por lo que se debe poner en marcha una matriz de planificación de estrategias que contengan los siguientes parámetros:

Estrategia: es un plan mediante el cual se busca lograr una meta a cumplir, para lo cual se empleará los 5 principios verdes analizados en el diseño del modelo.

Actividades: Es decir son las acciones que debe cumplir dentro de las empresas, de acuerdo a las estrategias planteadas.

Meta: Es el porcentaje (\%) que debe alcanzar para medir el éxito de una empresa y de cada una de sus actividades que realice.

Indicador: Mide el desempeño y el desarrollo de la gestión en base al cumplimiento de metas y el logro de objetivos deseados en un cierto tiempo.

Tiempo: Se debe organizar adecuadamente el tiempo y las estrategias a realizar, mientras dure el cambio para llegar a ser una empresa verde.

Responsable: Los encargados de realizar estos cambios dentro del modelo son los gerentes, administradores o propietarios, con apoyo si es necesario de un equipo de especialistas que tengan conocimientos en temas empresariales con base a emprendimientos verdes.

Presupuesto: Son los recursos monetarios que cuenta la empresa para lograr el cambio hacia un modelo de emprendimientos verde.

\section{4.- Evaluación y seguimiento}

Después de realizar la planificación, diseño y formulación, y ejecución dentro del modelo, donde se han identificado las deficiencias y debilidades en cada una de las etapas, es decir; se puede evaluar cada una de las etapas si tiene la aceptación deseada de acuerdo a las necesidades y falencias de cada uno de las Pymes cuencanas, caso contrario se debe buscar y combatir los errores que vayan surgiendo si el resultado no es el esperado.

Para lo cual se debe seguir ciertos pasos para poder llevar una mejor evaluación de las etapas:

- Se debe realizar un seguimiento y emitir informes si es necesario, en el cual se deben detallar los resultados obtenidos, nuevos hallazgos, decisiones tomadas para aplacar estas situaciones que aquejan a la empresa para lo cual se pueden utilizar gráficas y escritos de manera formal. Se puede realizar cada 3 meses este tipo de seguimientos e informes. 
- Se debe plantear indicadores que sirvan para monitorear los avances, logros obtenidos dentro de la empresa, por lo que la técnica a ser utilizadas para la medición y la recolección de los datos dentro del modelo será la técnica de semaforización, es decir, si está dentro del rango de 0\% - 40\% rojo, de 40\%-70\% está en amarillo y de $70 \%-100 \%$ verde, donde verde es equivalente a que la empresa está aplicando el modelo para convertirse en empresa o emprendimiento verde y que rojo, amarillo deben seguir trabajando para llegar a cumplir la meta en transformarse en semáforo verde.

\section{Conclusiones.}

- Se ha revisado la parte teórica sobre el emprendimiento verde que se basa en crear un negocio bajo los parámetros de conciencia ambiental dejando a atrás el modelo tradicional de destrucción de la naturaleza, se le puede llamar de diferentes nombres como: emprendimientos de base ecológica, eco-emprendimiento, emprendimiento sostenible, emprendimiento ecológico y emprendimiento sostenible y ambiental.

- El análisis de algunos modelos de emprendimientos exitosos a nivel internacional, es muy importante para conocer las realidades y los impactos que genera las empresas en el mundo y cuáles serían los cambios que se pueden realizar para aplicarlos en un determinado lugar.

- El modelo de gestión para emprendimientos verdes propuesto, se fundamenta en 4 etapas que se clasifican en identificación, diseño, ejecución, evaluación y seguimiento; para lo cual se consideran los 5 principios verdes que ayudan a favorecer a la sostenibilidad empresarial. Considerando lo anterior se detallan cada una de las etapas en la primera etapa se encuentran los problemas que cuenta la empresa, en la segunda se diseñan las estrategias aplicando los 5 principios verdes, en la tercera se ejecuta el diseño por medio de una matriz de planificación de estrategias y por último se evalúa de acuerdo a la técnica de semaforización donde se analiza el porcentaje de avance de las empresas para lograr catalogarse empresas verdes.

- Se considera este modelo como una herramienta eficaz aplicable a todo tipo de empresa sin importar su tamaño o sector económico, minimizando los impactos negativos generados en la sociedad y el medio ambiente, generando un desarrollo sostenible en el tiempo. El cual esta validado por 3 expertos, en donde se obtuvo que el modelo propuesto es factible, pertinente, aplicable y cuenta con un aporte teórico sustancial, y puede ser implementado en cualquier tipo de empresas.

\section{Referencias bibliográficas.}

Amate, J. (2015). Como hacer rentable el compromiso con la sostenibilidad de su empresa. Sostenibilidad a medida, 4-7. 
Arroyo, F., Bravo, D., Buenaño, C., \& Villarreal, F. (2018). La Innovación Verde Del Modelo De Negocio. INNOVA Research Journal, 109-111.

Barcellos, L. (2010). Modelos de gestion aplicados a la sostenibilidad empresarial. Barcelona: Universitat de Barcelona.

Baumol, W. (1968 y 1993). The Entrepreneur in Economic Theory. American Economic Review; Boston: MIT Press.

Braudel, F. (1985). La dynamique du capitalisme. Champs historie.

CAF. (2015). Modelo de gestión empresarial para micro y pequeñas empresas basadas en Negocios Verdes. Retrieved from http://scioteca.caf.com/handle/123456789/684

Casson, M. (1982). The entrepreneurs: an economic theory. Nueva . Nueva York: Barnes and Noble.

Chaves, M., \& Castro, R. (2015). Entrepreneurship as an aim of the European Union policy for higher education. Educação e Pesquisa, 513-526.

CLDR. (2019). POBREZA Y DESIGUALDAD INFORME LATINOAMERICANO. Rimisp - Centro Latinoamericano para el Desarrollo Rural, Centro Latinoamericano para el Desarrollo Rural. Chile: Fyrma. Retrieved 07 01, 2020, from https://rimisp.org/informelatinoamericano/wpcontent/uploads/2020/04/Rimisp-Informe-Latam-2019.pdf

ComunicarSe. (2018). comunicarseweb. Retrieved from https://www.comunicarseweb.com/noticia/60-empresas-adoptan-principios-deeconomia-verde-de-la-bolsa-de-valores

Crespo, J. (2018). Urban Bike Bilbao: mucho más que una gran tienda de bicis. Revista Ciclosfera mas bien, mejores ciudades.

Dean, T., \& McMullen, J. (2007). Toward a theory of sustainable entrepreneurship: Reducing environmental degradation through entrepreneurial action. Journal of Business Venturing, 50-76.

Delgadillo, J., \& Alburquerque, F. (2010). Emprendimientos de base ecológica, un modelo de interacción económica y territorial en Áreas Naturales y Protegidas de España y México. Mexico: 1a ed., Instituto de Investigaciones EconómicasUNAM, Universidad de Sevilla, Colegio de Tlaxcala, México.

Dolabela, F. (2010). Pedagogía Emprendedora.

EKOS . (2012). Informe Empresas Responsables Ecuador 2012. Ekos Negocios, 70-73.

Escuela Europea de Excelencia. (2019, 05 27). NUEVAS NORMAS ISO ES UNA INICIATIVA DE ESCUELA EUROPEA DE EXCELENCIA. Retrieved from 
https://www.nueva-iso-14001.com/2019/05/empresas-verdes-definicion-yanalisis/

Groncol. (2016). Infraestrutura Verde. Retrieved from http://groncol.com/blog/

Gunawan, A. (2014). Preliminary Study of Classifying Indonesian Entrepreneurs. Procedia - Social and Behavioral Sciences, 243-250.

Hancioglu, Y., Uslu, Y., \& Demir, E. (2015). Applicability to Green Entrepreneurship in Turkey: A Situation Analysis. ScienceDirect, 1238-1245.

Koga, R. (2016). UN Global Compact releases a guide to Corporate Sustainability. BERKELEY MDP, 1.

Kuckertz, A., \& Wagner, M. (2010). The Influence of Sustainability Orientation on Entrepreneurial Intentions-Investigating the Role of Business Experienc. Journal of Business Venturing, 524-539.

Kurtagić, S., Silajdzic, I., \& Vucijak, B. (2015). Green entrepreneurship in transition economies: A case study of Bosnia and Herzegovina. Journal of Cleaner Production, 376-384.

Knight , F. (1921). Enterprise and profit. En Risk, uncertainly and profit. Chicago: University of Chicag, 264-290.

Lasio, V., Amaya, A., Zambrano, J., \& Ordeñana, X. (2020, 07 17). Global Entrepreneurship Monitor Ecuador 2019/2020. Retrieved from https://www.gemconsortium.org/report/gem-ecuador-20192020-report

Lasio, V., Ordeñana, X., Caicedo, G., Samaniego, A., \& Izquierdo, E. (2017). Global Entrepreneurship Monitor.

Manuel, G. (2015). Perdurabilidad, sostenibilidad empresarial y grupos de interés. Gestión de la sostenibilidad en el marco de las organizaciones, 14-49.

Moreno, Z., Olmos, H. M., \& Espirítu, R. (2010). Análisis de las características del emprendimiento y liderazgo en los países de Asia y Latinoamérica. Revista mexicana de estudios sobre la cuenca del Pacífico, 104-105.

Nicuesa, M. (2016, 11 29). Qué es el emprendimiento verde. Retrieved from https://empresariados.com/que-es-el-emprendimiento-verde/

OECD. (2011, 05 26). Entrepreneurship at a Glance 2011. Retrieved from https://doi.org/10.1787/9789264097711-en

Ojeda, E., \& Rodríguez, A. (2011). El Ecosistema Nacional de Emprendimiento.

Pérez, L. (2019, 07 08). ¿Cuál es la importancia de las alianzas estratégicas entre empresas? Retrieved from https://blogs.unitec.mx/vida- 
universitaria/emprendedores/la-importancia-de-las-alianzas-estrategicas-entreempresas

Ramírez, H. (2020). Transparencia empresarial: Genera confianza en tu empresa. Grupo Atico34, 1 .

Recalde , L., Villota , L., \& Flores, M. (2016). Emprendedores como creadores de riqueza y desarrollo regional. Revista Publicando, 564-478.

Salgado, S. (2012). La empresa debe delinear sus politicas y valores de acuerdo la filosofia de RSE. Revista Ekos, 71-72.

Schumpeter, J. (1935). The Analysis of Economic Change. Review of Economic Statistics Vol. XVII, 2-10.

Segura, J., \& Sanchez, M. (2003). PROPUESTA: ESTRATEGIAS DE DESARROLLO. $V$ SEMINARIO NACIONAL DE LA RED DE CENTROS ACADEMICOS PARA. Mendoza - Argentina.

Tejada, J. M. (2004). Administración de la calidad: Prácticas organizacionales percibidas y el compromiso de los trabajadores hacia la organización. Revista Interamericana de Psicologia Ocupacional.

Torre, A. G. (2018). LA TRANSICIÓN HACIA UNA ECONOMÍA BAJA EN CARBONO: UNA OPORTUNIDAD PARA EL CLIMA Y EL EMPLEO. RUMBO 2030, (pp. 2 - 5).

Udiz, G. (2010). ¿Qué es el "conocimiento empresarial"? . Pymes y Autónomos, 1.

Vazquez, D., Smith , A., \& Sarkis, J. (2014). Managing the transition to critical green growth: The 'Green Growth State'. ScienceDirect, 38-50.

Yepes, M. (2012). ¿El emprendedor nace o se hace? Reflexiones sobre Emprendimiento.

Zahedi, A., \& Otterpohl, R. (2015). Towards Sustainable Development by Creation of Green Social Entrepreneur's Communities. ScienceDirect, 196-201.

Zoninsein, L., Cesano, D., \& Russell, J. (2014). Economía Verde. Lima: Soluciones Prácticas.

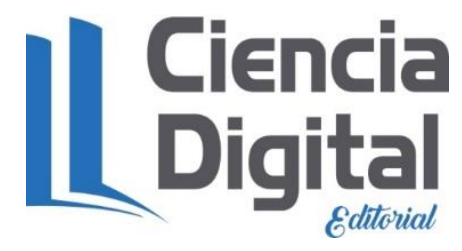




\section{PARA CITAR EL ARTÍCULO INDEXADO.}

Plaza León, I. D., Banegas Campoverde, C. M., \& Castillo Ortega, Y. (2021). Los emprendimientos verdes una alternativa de sostenibilidad para las pymes en la ciudad de
Cuenca.
Visionario
Digital,
$5(2)$,
6-29.

https://doi.org/10.33262/visionariodigital.v5i2.1633

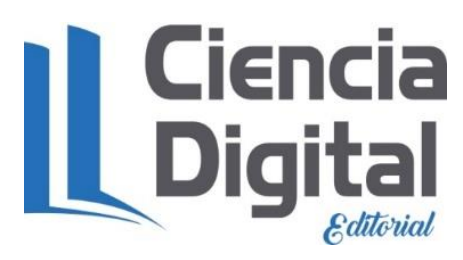

El artículo que se publica es de exclusiva responsabilidad de los autores y no necesariamente reflejan el pensamiento de la Revista Visionario Digital.

El artículo queda en propiedad de la revista y, por tanto, su publicación parcial y/o total en otro medio tiene que ser autorizado por el director de la Revista Visionario Digital.
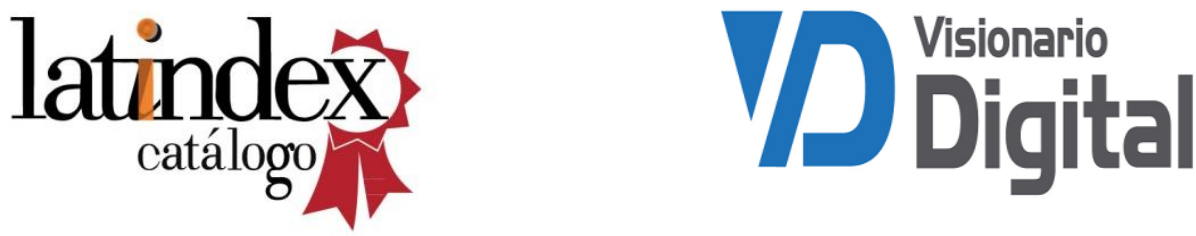